\title{
Strained Tetragonal States and Bain Paths in Metals
}

\author{
P. Alippi, ${ }^{1}$ P. M. Marcus, ${ }^{1,2}$ and M. Scheffler ${ }^{1}$ \\ ${ }^{1}$ Fritz-Haber-Institut der Max-Planck-Gessellschaft, Faradayweg 4-6, D-14195 Berlin-Dahlem, Germany \\ ${ }^{2}$ IBM Research Center, Yorktown Heights, New York 10598
}

(Received 24 September 1996)

\begin{abstract}
Paths of tetragonal states between two phases of a material, such as bcc and fcc, are called Bain paths. Two simple Bain paths can be defined in terms of special imposed stresses, one of which applies directly to strained epitaxial films. Each path goes far into the range of nonlinear elasticity and reaches a range of structural parameters in which the structure is inherently unstable. In this Letter we identify and analyze the general properties of these paths by density functional theory. Special examples include vanadium, cobalt, and copper, and the epitaxial path is used to identify an epitaxial film as related uniquely to a bulk phase. [S0031-9007(97)03190-6]
\end{abstract}

PACS numbers: $64.70 . \mathrm{Kb}, 61.50 . \mathrm{Ks}, 62.20 . \mathrm{Dc}$

Pseudomorphic epitaxy of a cubic or tetragonal (001) film typically results in a strained tetragonal structure. If the stresses on the tetragonal state vanish and also the state corresponds to a local minimum of energy with respect to tetragonal deformations, the structure will be called a tetragonal phase. Such a phase will be stable or metastable, depending on whether it has the lowest energy compared to other minima. Frequently metals have two tetragonal phases; sometimes both are cubic, e.g., bcc and fcc $\mathrm{Na}$ and $\mathrm{Rb}[1,2]$; sometimes one is cubic and the other phase is tetragonal, e.g., Ti and V [2,3]. They can, of course, also have phases with other structures; e.g., Ti also has a hcp phase.

Many paths can go from one tetragonal phase to the other. If the geometries along such a path have tetragonal symmetry and if they connect bcc and fcc phases, the paths have been called Bain paths [4]. A purpose of the present work is to define and discuss a particular Bain path which will be called the epitaxial Bain path (EBP). The EBP is produced by isotropic stress or strain in the (001) plane of tetragonal phases accompanied by vanishing stress perpendicular to the plane, such as pseudomorphic epitaxy produces on an (001) cubic or tetragonal film. Epitaxy provides a valuable means of stabilizing metastable phases and of putting phases under very large strains, both tensile and compressive in the plane of the epitaxy. The EBP of a material identifies the phase that has been strained, checks quantitatively the elastic behavior, which can be highly nonlinear, and predicts which phase of the material will form on a given substrate. Thus, in order to understand the properties of epitaxial films and new materials, the knowledge of the EBP is indispensable.

A different Bain path has long been discussed, particularly by Milstein [1], in which uniaxial stress is applied to a tetragonal state along the [001] axis accompanied by zero stress in the (001) plane; this path is conveniently called the uniaxial Bain path (UBP). We compare the two paths, EBP and UBP, which are both physically realizable. We show that both have the same lowest possible maximum energy or barrier energy of all Bain paths between the two tetragonal phases. However, the EBP has a special value in relating strained tetragonal structures produced by epitaxy to particular tetragonal phases. Depending on where the tetragonal structure lies on the EBP, we shall show that a clear choice can be made which classifies the structure as a strained form of a particular phase. Thus for materials with both a bcc and a fcc phase, the bulk (i.e., the interior) of an epitaxial film can be uniquely classified as a strained bcc phase or a strained fcc phase. Experimental geometry analyses of the bulk structure are frequently made by low-energy electron diffraction of ultrathin epitaxial films, which can then be placed on the EBP in the tetragonal plane to identify the phase of which it is the strained form.

We employ density functional theory together with the full-potential linearized augmented plane wave method [5]. An important advantage of a first-principles calculation, i.e., a calculation with no adjustable parameters, is that it applies just as reliably to highly strained or even unstable geometries, as it does to the unconstrained ground state, i.e., to the stable phase. The same reliability cannot be asserted for empirically adjusted potentials, such as the empirical pseudopotential model used in Ref. [1], which is adjusted to reproduce ground-state properties of only certain structures. The first-principles calculation provides a check on the validity of empirical potentials far from the structural ground state and of linear elasticity theory.

The calculation proceeds by evaluating the total energy $E(a, c)$ in $\mathrm{eV} /$ atom as a function of the tetragonal lattice constants $a$ and $c$. Contour lines of constant $E$ are plotted in Fig. 1 for vanadium on the $\left(a / a_{\mathrm{bcc}}-V / V_{\mathrm{bcc}}\right)$ plane, where $V=c a^{2} / 2$ is the volume per atom. The contour lines show clearly two minima, one at the bcc structure and one at a tetragonal structure, and between them a saddle point at the fcc structure. Thus vanadium has two fairly deep and well-separated minima and so is a good choice to illustrate the tetragonal paths between 


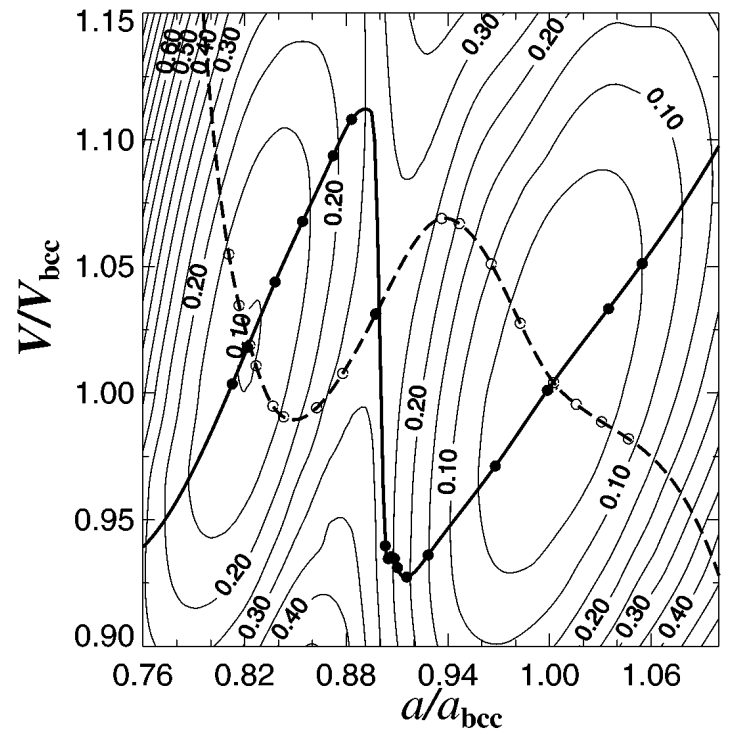

FIG. 1. Contour lines of constant $E$ in $\mathrm{eV} /$ atom for vanadium on the tetragonal plane showing a bcc minimum (the zero of $E$ ) with $a_{\mathrm{bcc}}=2.93 \AA$ and $V_{\mathrm{bcc}}=12.6 \AA^{3}(c / a=1)$, a minimum at $a=2.41 \AA(c / a=1.83)$ and $V=12.8 \AA^{3}$, and a saddle point at $a=2.65 \AA\left[c / a=(2)^{1 / 2}\right]$ and $V=13.1 \AA^{3}$. The epitaxial Bain path (EBP, full dots) and the uniaxial Bain path (UBP, open dots) are also shown.

minima [6]. Since $E$ has an extremum at the cubic structures [3,7], the saddle point will occur exactly at the fcc structure.

Also plotted in Fig. 1 are the EBP and the UBP. The EBP is calculated from the minima of $E(c)$ at constant $a$, which corresponds to the epitaxial situation, i.e., $a$ is held fixed and $c$ adjusts to minimize $E(c)$ and thus makes the out-of-plane stress, i.e., the stress along [001], vanish. Conversely, the UBP calculation fixes $c$ and lets $a$ adjust to minimize $E(a)$, making the in-plane stress vanish. Both paths go through the minima and the saddle point, hence the maximum energy on each path is the same.

The saddle point is called in Ref. [1] "a special unstressed tetragonal state ... at a local energy maximum" [8]. Obviously any path on the tetragonal plane that does not go through the saddle point must go through states of higher energy than the saddle point, since it will cross contours in either the upper or lower central sectors formed by the saddle-point contours at $E=0.29 \mathrm{eV} /$ atom. Two common paths that do not go through the saddle point, hence have higher maximum energies than the EBP or UBP, are as follows: (1) The constant volume paths at the volumes of the minima, which differ from the saddle-point volume. These paths have been called volume conserving Bain paths $[2,3]$. (2) The hydrostatic pressure path, which has equal in-plane and out-of-plane stress components.

Figure 2 plots $E$ vs $a$ along the EBP and UBP. Also indicated is the range from $a=2.58 \AA(c / a=1.6)$ to $a=2.66 \AA(c / a=1.2)$ for which we find along the EBP

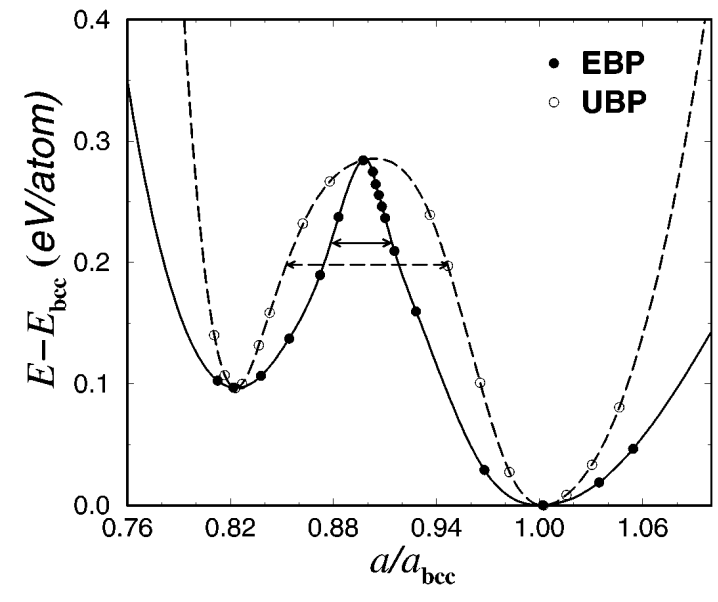

FIG. 2. Plots of $E$ in $\mathrm{eV} /$ atom along the Bain paths EBP and UBP for vanadium between the minima and through the saddle point. The unstable ranges of $a$ are marked with arrows for both paths.

the tetragonal structure to be inherently unstable [9]. In this range the slope of the EBP changes sign, and the stability condition

$$
\tilde{c}_{11} \tilde{c}_{33}-\tilde{c}_{13}^{2} \geq 0
$$

is violated. Here $\tilde{c}_{i j}$ are calculated from $\tilde{c}_{11}=\left(a^{2}\right)$ $V) \partial^{2} E(a, c) / \partial a^{2}, \quad \tilde{c}_{13}=(a c / V) \partial^{2} E(a, c) / \partial a \partial c, \tilde{c}_{33}=$ $\left(c^{2} / V\right) \partial^{2} E(a, c) / \partial c^{2}$ for the strained lattice [10]. Tetragonal states outside the range of Eq. (1) cannot be stabilized with any applied stresses. When a path on the tetragonal plane reaches the boundaries of this unstable region, the tetragonal crystal becomes unstable and an epitaxial film will not be able to form many layers at the bulk spacing. Epitaxial films strained to a state near the unstable region should show large changes in the phonon spectra as elastic constants weaken, which would be interesting to study.

One consequence of the existence of an unstable region is that a clear conceptual distinction can be made between strained states of the phase at lower $c / a$ (for vanadium the bcc phase) and strained states of the larger $c / a$ phase (for vanadium at $c / a=1.83$ ). If there was a continuous path of allowed states between the two phases, there would not be a clear criterion for regarding an observed strained state as strained from a particular one of the two phases.

A second consequence of the unstable region is that the strained system never gets to the saddle point, but transforms to another strained phase at an energy at or below the energy of the state where the path strikes the boundary of the unstable region. Thus for the EBP and UBP the breakdown energy is at least $0.05 \mathrm{eV} /$ atom lower than the saddle-point energy, and it is lower on the UBP.

The EBP and UBP can be found for other metals by similar calculations; for $\mathrm{Cu}$ and $\mathrm{Co}$ our results are shown in Fig. 3 and Fig. 4. The topology of the $E(a, c)$ surface 




FIG. 3. Epitaxial Bain paths on the $\left(a / a_{\mathrm{fcc}}-V / V_{\mathrm{fcc}}\right)$ plane for Co, with LEED bulk structures for strained $\mathrm{Co} / \mathrm{Rh}(001)$ [13], $\mathrm{Co} / \mathrm{Cu}(001)$ [14], and $\mathrm{Co} / \mathrm{Fe}(001)$ [15]. The error limits are shown by a line through the point and come entirely from the estimated error in $c$ from experimental analysis. The theoretical value of $a_{\mathrm{fcc}}$ for the fcc phase is $2.50 \AA\left(V_{\mathrm{fcc}}=11.09 \AA^{3}\right)$, which agrees with the experimental value [16]. The dot-dashed lines are obtained from linear elasticity theory, with elastic constants of the fcc structure. The horizontal arrow marks the range along the EBP path where Eq. (1) is violated.

of cobalt is similar to the one of vanadium, with the two minima and the saddle point corresponding to the structures where the EBP and the UBP intersect. In this case, however, the stable cubic phase is face-centered $(c / a=$ $\sqrt{2})$ with $a_{\mathrm{fcc}}=2.50 \AA$, the bcc structure corresponds to the saddle point $\left(a_{\mathrm{bcc}}=2.83 \AA\right)$, and a metastable tetragonal structure is found at $c / a=0.92(a=2.91 \AA)$ [11]. The energy barrier $E_{\mathrm{bcc}}-E_{\mathrm{fcc}}$ is $72 \mathrm{meV}$.

The situation is different for $\mathrm{Cu}$ : The stable phase is at the fcc structure $\left(a_{\mathrm{fcc}}=2.53 \AA\right)$, but there is no

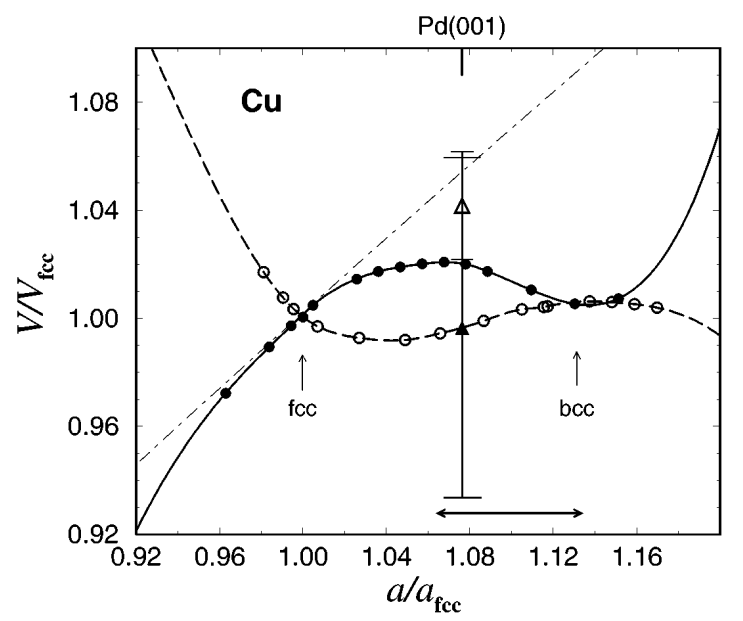

FIG. 4. Same as previous figure, but for $\mathrm{Cu}$. The LEED [17] (open triangle) and the STM [18] (full triangle) bulk results for $\mathrm{Cu} / \mathrm{Pd}(001)$ are given as well. The theoretical value of $a_{\mathrm{fcc}}$ for $\mathrm{Cu}$ is $2.53 \AA\left(V_{\mathrm{fcc}}=11.52 \AA^{3}\right)$, while experimental points use the experimental value of $a_{\mathrm{fcc}}=2.56 \AA\left(V_{\mathrm{fcc}}=11.76 \AA^{3}\right)$. other tetragonal metastable phase, and the energy surface $E(a, c)$ shows a large plateau near the bcc structure [12]; for this reason, the EBP and the UBP share a common part for $1.13 \leq a / a_{\mathrm{fcc}} \leq 1.15$.

Strained structures found by LEED are entered in the graphs; in each case the error limits intersect the EBP. Prediction of which phase will be produced and how strained it will be by a given substrate can be immediately made by entering the $a$ of the substrate on the $a$ axis of the EBP.

For $\mathrm{Co}$ on $\mathrm{Rh}(001)$ [13] and on $\mathrm{Cu}(001)$ [14] the structures lie on the left of the unstable region and thus both correspond to strained fcc structures. However, the error line for $\mathrm{Co}$ on $\mathrm{Fe}(001)$ [15] is at a value of $a$ above the unstable range and must be considered a strained form of the tetragonal phase of Co at $c / a=0.92$; bcc Co itself at $c / a=1(a=2.83 \AA)$ is unstable.

The strained tetragonal structure for $\mathrm{Cu}$ on $\mathrm{Pd}(001)$ lies in the range of the EBP identified as unstable by our theory. We therefore disagree with the conclusions of a recent scanning tunneling microscopy (STM) experiment [18], where such a film was "identified" as a metastable tetragonal phase. The structure measured in the high coverage regime (1-7 layers at $T=300 \mathrm{~K}, 3-7$ layers at higher temperatures) agreed within experimental error with the LEED analysis [17]; however, the morphology of the islands observed in the STM pictures was taken as indication of a structure under small planar strain, and, therefore, of the existence of a $\mathrm{Cu}$ tetragonal phase with lattice constant close to $a_{P d}$. Our results predict that there is no tetragonal phase at $c / a<\sqrt{2}$ (and $a>a_{\mathrm{fcc}}$ ); we also show that the film is too far from the bcc region of the EBP to be regarded as strained bcc. Thus, we conclude that the properties of such $\mathrm{Cu} / \mathrm{Pd}(001)$ film are not bulk like, but instead stabilized by the (still ultrathin) film properties.

In Fig 4 (as in Fig. 3) the epitaxial paths from linear elasticity theory are also drawn for fcc $\mathrm{Cu}$ (and $\mathrm{Co}$ ). Epitaxial strain on a cubic (001) surface in the linear elastic approximation says normal stress vanishes, hence $\delta c / c=-[2 \nu /(1-\nu)] \delta a / a$, where $\nu$ is the Poisson ratio. A comparison with the calculated EBP in both figures shows how the linear approximation fails to be valid for $\delta a / a$ equals to few percent. In the analysis of the $\mathrm{Cu} / \mathrm{Pd}(001)$ film, it is evident that the film is already far from the linear elastic regime.

Generalizations of this calculation of physically realizable paths between phases are possible with existing codes. Milstein [19] has considered the instability of tetragonal structures with respect to orthorhombic deformations and found such instabilities on the UBP outside the range of $c / a$ between the two minima; these instabilities probably exist on the EBP also, but have not as yet been studied. It looks practical to explore with present codes even more general paths of homogeneous strain between tetragonal phases that make more general deformations of 
the unit mesh than orthorhombic deformations. Possibly a path of deformed lattices could be found with lower maximum energies, as the path of trigonal lattices of Ref. [7]. It would also be possible to find epitaxial paths of tetragonal states for slabs of a finite number of layers, which would include relaxations of surface layers.

In summary, paths of tetragonal states between two tetragonal phases include two particularly simple cases, namely, those produced by epitaxial (isotropic biaxial) stress and by uniaxial stress. Both cases correspond to maintaining certain surfaces stress-free, and both have minimal maximum energies at a saddle point of energy that both pass through. Along these paths a range of instability exists, which separates states that can be clearly regarded as strained from one or the other of the two phases. The epitaxial path permits definite identification of the equilibrium phases of strained epitaxial films and could usefully be found for all metals with tetragonal phases.

[1] Frederick Milstein, Huei Fang, and Jochen Marschall, Philos. Mag. A 70, 621 (1994).

[2] V.L. Sliwko, P. Mohn, K. Schwarz, and P. Blaha, J. Phys. Condens. Matter 8, 799 (1996).

[3] P. J. Craievich, M. Weinert, J. M. Sanchez, and R.E. Watson, Phys. Rev. Lett. 72, 3076 (1994).

[4] E. C. Bain, Trans. Am. Inst. Min. Metall. Eng. 70, 25 (1924).

[5] P. Blaha, K. Schwarz, and R. Augustyn (unpublished); P. Blaha, K. Schwarz, and S. B. Trickey, Comput. Phys. Commun. 59, 399 (1990).

[6] A plot of energy contour lines for vanadium is also given in Ref. [2], which provided in addition a useful power series expansion of $E$ with which the present calculations were checked.

[7] T. Kraft, P. M. Marcus, M. Methfessel, and M. Scheffler, Phys. Rev. B 48, 5886 (1993).

[8] The saddle point is not actually a local maximum of energy, since $E$ increases from that point in the central sectors formed by the saddle-point contours at $E=$ $0.29 \mathrm{eV} /$ atom in Fig. 1.

[9] At all $V$ in Fig. 1, the unstable region boundaries have about the same $c / a$ values.

[10] J.F. Nye, Physical Properties of Crystals (Clarendon, Oxford, 1957). This condition, along with $\tilde{c}_{11} \geq 0$, is the condition that the energy of the strained tetragonal crystal (held in equilibrium by applied stresses) is positive definite with respect to tetragonal deformations. The elastic constants $\tilde{c}_{i j}$ correspond to tetragonal deformations in which the constraint $a_{1}=a_{2}$ is applied. They are related to the usual elastic constants by $\tilde{c}_{11}=2\left(c_{11}+\right.$ $\left.c_{12}\right), \tilde{c}_{13}=2 c_{13}, \tilde{c}_{33}=c_{33}$.

[11] This result for bcc Co is in agreement with the work of Amy Y. Liu and David J. Singh, Phys. Rev. B 47, 8515 (1993).

[12] This is in agreement with previously reported calculations [7]. Recently Jeong [Sukmin Jeong, Phys. Rev. B 53, 13973 (1996)] has found a tetragonal metastable phase at $c / a=0.95$, but with a small energy barrier, which is of the same order of the numerical accuracy of the calculations; he has also calculated the point on the EBP for $\mathrm{Cu}$ at the $\mathrm{Pd}$ lattice constant.

[13] A. M. Begley, S. K. Kim, F. Jona, and P. M. Marcus, J. Phys. Condens. Matter 5, 1 (1993).

[14] A. Clarke, G. Jennings, R. F. Wells, P. J. Rous, and J. B. Pendry, Surf. Sci. 187, 327 (1987); J. R. Cerdá, P. L. de Andres, A. Cebollada, R. Miranda, E. Navas, P. Schuster, C. M. Schneider, and J. Kirschner, J. Phys. Cond. Matter 8, 2055 (1993).

[15] S. K. Kim, C. Petersen, F. Jona, and P. M. Marcus, Phys. Rev. B 54, 2184 (1996).

[16] J. Donohue, The Structures of the Elements (Wiley, New York, 1974).

[17] H. Li, S. C. Wu, D. Tian, J. Quinn, Y.S. Li, F. Jona, and P. M. Marcus, Phys. Rev. B 40, 5841 (1989).

[18] E. H. Hahn, E. Kampshoff, N. Wälchli, and K. Kern, Phys. Rev. Lett. 74, 1803 (1995).

[19] Frederick Milstein, Jochen Marschall, and Huei Eliot Fang, Phys. Rev. Lett. 74, 2977 (1995). 